Pacific Journal of Mathematics

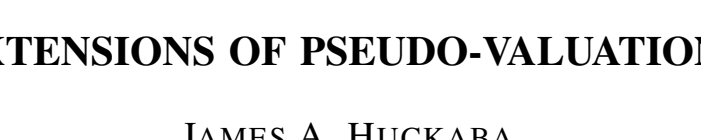




\section{EXTENSIONS OF PSEUDO-VALUATIONS}

\section{JAMES A. HuCKabA}

Let $w$ be a pseudo-valuation defined on a commutative ring $R$ and let $S$ be an overring of $R$. This paper investigates conditions needed to imply that $w$ can be extended to $S$. These conditions are given in terms of a particular sequence of ideals $\left\{A_{i}\right\}_{i=0}^{\infty}$ in $R$ which is called the best filtration for $w$. The main theorem states that if $w$ is a pseudo-valuation on $R$ with best filtration $\left\{A_{i}\right\}$ and each $A_{i}$ is a contracted ideal with respect to $S$, then $w$ can be extended to $S$. The converse of this result is then proved.

By using our main theorem and some recent results by Gilmer [1], we show in several important cases that if $S$ is an overring of $R$ and $w$ is any pseudo-valuation on $R$ possessing a best filtration, then $w$ can be extended to $S$. In particular, if $R$ is a Prüfer domain with quotient field $K$ and if $S$ is an overring of $R$ such that $S \cap K=R$, then $w$ can be extended from $R$ to $S$.

We begin in $\S 1$ by defining and developing properties of a best filtration and determining classes of pseudo-valuations which have best filtrations. The main results and applications are then proved in $\S 2$.

1. Filtrations. All rings are commutative, associative, and have identity. If $S$ is an overring of $R$, we assume that $S$ and $R$ have the same identity. A pseudo-valuation on the ring $R$ is a mapping $w$ from $R$ into the extended real number system such that:

(i ) $w(0)=\infty, w(1)=0$,

(ii) $w(x-y) \geqq \min \{w(x), w(y)\}$,

(iii) $w(x y) \geqq w(x)+w(y)$, for each $x, y \in R$.

$w$ is called a homogeneous pseudo-valuation in case:

(iv) $w\left(x^{2}\right)=2 w(x)$ for each $x \in R$.

$w$ is called a valuation in case:

(v) $w(x y)=w(x)+w(y)$ for each $x, y \in R$.

Pseudo-valuations were first introduced by Rees [3]. Rees proved in [3] that (iv) is equivalent to the condition that $w\left(x^{n}\right)=n w(x)$ for each positive integer $n$ and for each $x \in R$. These functions arise quite naturally in ring theory. If $A$ is a proper ideal of $R$, define $v_{A}(x)=n$ if $x \in A^{n}, x \notin A^{n+1}$ and $v_{A}(x)=\infty$ if $x \in A^{n}$ for all $n$. Then $v_{A}$ is a pseudo-valuation. We say that $v_{A}$ is associated with the ideal $A$. A sequence of ideals $\left\{A_{i}\right\}_{i=0}^{\infty}$ of $R$ such that $A_{0}=R, A_{i+1} \subset A_{i}$, and $A_{i} A_{j} \subset A_{i+j}$ for all $i$ and $j$ is called a filtration on $R$. Notice that the nonnegative integral powers of an ideal $A$ of $R$ forms a filtration, 
where $A^{\circ}$ is defined to be $R$. Also note that any filtration $\left\{A_{i}\right\}$ determines a pseudo-valuation in exactly the same manner that the powers of an ideal $A$ determines $v_{A}$. For an arbitrary pseudo-valuation $w$ on $R$ and a subset $T$ of $R$, define $w(T)=\inf \{w(t): t \in T\}$.

Definition 1. If $w$ is a pseudo-valuation on $R$, define

$$
\begin{aligned}
A_{0}= & R \\
A_{i}= & \left\{x \in R: w(x)>w\left(A_{i-1}\right)\right\}, \\
& \text { if } w\left(A_{i-1}\right)<\infty . \\
A_{i}= & A_{i-1} \text {, if } w\left(A_{i-1}\right)=\infty .
\end{aligned}
$$

Each member of the sequence $\left\{A_{i}\right\}$ is an ideal of $R$. The sequence defined by (1.1) has the property that $A_{0} \supset A_{1} \supset A_{2} \supset \cdots . A_{i+1}$ is not necessarily a proper subset of $A_{i}$, as will be shown in Examples 1 and 2. Also note that for a given pseudo-valuation $w,\{x \in R: w(x)=$ $\infty\} \subset \cap A_{i}$. The following example shows that there exists pseudovaluations such that the sequence defined by (1.1) is not a filtration.

EXAMPLE 1. Let $\mathfrak{A}$ be an ideal of a ring $R$ in which $\mathfrak{A}^{i} \supsetneq \mathfrak{A}^{i+1}$ for all $i$. Define a sequence of ideals $\left\{B_{i}\right\}$ as follows: $B_{0}=R, B_{1}=\mathfrak{A}^{2}$, $B_{2}=\mathfrak{A}^{3}, B_{3}=B_{4}=\mathfrak{U}^{5}$, and $B_{i}=\mathfrak{A}^{7}(i \geqq 5)$. Then $\left\{B_{i}\right\}$ is a filtration in $R$ and determines some pseudo-valuation $w$, where $w(x)=n$ if $x \in \varepsilon B_{n}, x \notin B_{n+1}$ and $w(x)=\infty$ if $x \in \cap B_{n}$. Now use Definition 1 to define $A_{i}$ with respect to $w$. We obtain $A_{i}=B_{i}(i=0,1,2,3)$ and $A_{i}=$ $B_{i+1}(i=4,5, \cdots)$. But $\left\{A_{i}\right\}$ is not a filtration, since $\left(A_{2}\right)^{2} \not \subset A_{4}$.

Definition 2. Let $w$ be a pseudo-valuation on $R$ and let $\left\{A_{i}\right\}$ be defined by (1.1). If $\left\{A_{i}\right\}$ is a filtration in $R$ such that $x \in \cap A_{i}$ if and only if $w(x)=\infty$, then $\left\{A_{i}\right\}$ is called a best filtration for $w$. Let $B(R)$ denote the class of all pseudo-valuations on $R$ which have a best filtration.

Example 1 then implies that there are pseudo-valuations which do not have best filtrations. It is clear from the definition that if $w$ has a best filtration, then it is unique. From now on we will talk about the best filtration for $w$.

EXAMPLE 2. Let $w$ be a pseudo-valuation on $R$ and let $\left\{A_{i}\right\}$ be the sequence defined by (1.1). It is possible for $\left\{A_{i}\right\}$ to be a filtration in $R$, yet not be the best filtration for $w$. Let $v$ be a real valued nondiscrete valuation on a field $K$ and consider $v$ as a pseudo-valuation on its valuation ring $R_{v}$. Since the value group of $v$ has no smallest positive element, $v\left(A_{1}\right)=0$. Then $A_{2}=\left\{x \in R: v(x)>v\left(A_{1}\right)=0\right\}=A_{1}$. 
By induction, we see that $A_{i}=A_{1}$ for each $i \geqq 1$. Hence the sequence $\left\{A_{i}\right\}$ is such that $A_{0} \supsetneq A_{1}=A_{2}=\cdots$. Therefore $v \notin B(R)$. However, it is clear that $\left\{A_{i}\right\}$ is a filtration.

We will be interested only when the sequence defined by (1.1) is a filtration. This always happens in one important case.

REMARK 1. If $v$ is a valuation on a ring $R$ and if $\left\{A_{i}\right\}$ is the sequence of ideals defined by (1.1), then $\left\{A_{i}\right\}$ is a filtration in $R$.

Proof. It is clear that $A_{i} \supset A_{i+1}$ for each $i$. Hence, to complete the proof we need to show that $A_{i} A_{j} \subset A_{i+j}$ for all nonnegative integers $i$ and $j$. We fix $j$ and use induction on $i$. Clearly $A_{0} A_{j} \subset A_{0+j}$. Assume that $A_{i-1} A_{j} \subset A_{i+j-1}$ for $i \geqq 1$. Let $x \in A_{i} A_{j}$, then $x=\sum_{k=1}^{n} a_{k} b_{k}$ where $a_{k} \in A_{i}$ and $b_{k} \in A_{j}$. We may assume without loss of generality that $v\left(a_{1}\right)+v\left(b_{1}\right)=\min _{k=1}^{n}\left(v\left(a_{k}\right)+v\left(b_{k}\right)\right)$. Then $v(x) \geqq v\left(a_{1}\right)+v\left(b_{1}\right)$. Case 1: If $v\left(A_{i-1}\right)<\infty$, then $v\left(a_{1}\right)>v\left(A_{i-1}\right)$, and thus $v(x)>v\left(A_{i-1}\right)+v\left(A_{j}\right)=$ $v\left(A_{i-1} A_{j}\right) \geqq v\left(A_{i+j-1}\right)$. By Definition 1, $x \in A_{i+j}$. Case 2: If $v\left(A_{i-1}\right)=\infty$, then $v(x)=\infty$, which implies that $x \in A_{i+j}$. Therefore $A_{i} A_{j} \subset A_{i+j}$.

LEMma 1. Let $w \in B(R)$ and let $\left\{A_{i}\right\}$ be the best filtration for $w$. Then:

(1) $A_{i}=A_{i+1}$ if and only if $w\left(A_{i}\right)=\infty$.

(2) Let $x \in A_{i}$ and $x \notin A_{i+1}$. Then $y \in A_{i}$ and $y \notin A_{i+1}$ if and only if $w(x)=w(y)$. In fact, $w(x)=w\left(A_{i}\right)$.

(3) If $y \in A_{i}$ and $z \notin A_{i}$, then $w(y)>w(z)$.

(4) If $w(x)<\infty$, then there exists an integer $i$ such that $x \in A_{i}$ and $x \notin A_{i+1}$.

Proof. (1) Suppose $A_{i}=A_{i+1}$. By induction we see that $A_{i}=A_{i+t}$ for each positive integer $t$. If $w\left(A_{i}\right)<\infty$, then there is an element $x \in A_{i}$ such that $w(x)<\infty$. But $x \in \cap A_{i}$ which implies that $w(x)=\infty$, a contradiction. Conversely, if $w\left(A_{i}\right)=\infty$, then $A_{i}=A_{i+1}$ by definition of the best filtration.

(2) First note that $x \in A_{i}, x \in A_{i+1}$ implies that $w(x)=w\left(A_{i}\right)$. If $y \in A_{i}, y \notin A_{i+1}$, then clearly $w(x)=w(y)$. Conversely, assume $w(x)=$ $w(y)$. If $i=0$, then $w(y) \leqq w\left(A_{1}\right)$ and hence $y$ is in $A_{0}$, but not in $A_{1}$. If $i>0$, then $w\left(A_{i-1}\right) \leqq w\left(A_{i}\right)$. If equality holds, then $A_{i}=A_{i+1}$, which implies that $x \in A_{i+1}$. Therefore $w\left(A_{i-1}\right)<w\left(A_{i}\right)$, which implies that $y \in A_{i}$. Also $y \notin A_{i+1}$, for if so, then $w(y)>w\left(A_{i}\right)$.

(3) and (4) are clear.

The converse of the above result is also true. 
Lemma 2. Let $w$ be a pseudo-valuation on $R$ and let $\left\{B_{i}\right\}$ be a filtration in $R$ satisfying properties (1)-(4). Then $\left\{B_{i}\right\}$ is the best filtration for $w$.

Proof. Clearly $x \in \cap B_{i}$ if and only if $w(x)=\infty$. Suppose that $w\left(B_{i-1}\right)<\infty$. By properties (2) and (3) $B_{i}=\left\{x \in R: w(x) \geqq w\left(B_{i}\right)\right\}$. Thus $B_{i} \subset\left\{x \in R: w(x)>w\left(B_{i-1}\right)\right\}$. On the other hand, suppose that $w(x)>w\left(B_{i-1}\right)$. If $w(x)=\infty$, then $x \in \cap B_{j}$ and hence $x \in B_{i}$. If $w(x)<\infty$, choose $k$ such that $x \in B_{k}$ and $x \notin B_{k+1}$. Suppose that $k \leqq i-1$, then $B_{k} \supset B_{i-1}$, so $w(x)=w\left(B_{k}\right) \leqq w\left(B_{i-1}\right)$, a contradiction. So we must have $k>i-1$ and hence $x \in B_{i}$. Therefore $B_{i}=$ $\left\{x \in R: w(x)>w\left(B_{i-1}\right)\right\}$.

By (1), if $w\left(B_{i-1}\right)=\infty$, then $B_{i-1}=B_{i}$.

We assume from now on that all pseudo-valuations $w$ which are considered have the property that there exists at least one $x$ such that $0<w(x)<\infty$.

Lemma 3. (a) If $w$ is a homogeneous pseudo-valuation on $R$ and if $\left\{A_{i}\right\}$ is the sequence of ideals defined by (1.1), then $w\left(A_{i}\right)<\infty$ for each $i$.

(b) If $w$ is a pseudo-valuation on a ring $R$ and if $\left\{A_{i}\right\}$ is the sequence of ideals defined by (1.1) such that each $A_{i}$ is finitely generated, then $w\left(A_{i-1}\right)<\infty$ implies that $w\left(A_{i}\right)>w\left(A_{i-1}\right)$.

Proof. (a) Suppose, to the contrary, that $i$ is the smallest positive integer such that $w\left(A_{i}\right)=\infty$. Since $w$ is nontrivial, $i \geqq 2$. Choose $x \in A_{i-1}, x \notin A_{i}$. Then $0<w(x)<\infty$, and $w\left(x^{2}\right)>w(x) \geqq w\left(A_{i-1}\right)$, so $x^{2} \in A_{i}$. But, $w\left(A_{i}\right) \leqq w\left(x^{2}\right)=2 w(x)<\infty$, contradicting the assumption that $w\left(A_{i}\right)=\infty$.

(b) Let $a_{1}, \cdots, a_{n}$ be a basis for $A_{i}$. Choose $a_{k}$ such that $w\left(a_{k}\right)=$ $\min \left\{w\left(a_{1}\right), \cdots, w\left(a_{n}\right)\right\}$. Then $w\left(A_{i}\right)=w\left(a_{k}\right)$. Since $a_{k} \in A_{i}, w\left(a_{k}\right)>w\left(A_{i-1}\right)$ and therefore $w\left(A_{i}\right)>w\left(A_{i-1}\right)$.

The following theorem shows that there are many pseudo-valuations with best filtrations.

THEOREM 1. (1) Any pseudo-valuation associated with an ideal is in $B(R)$. More generally, any pseudo-valuation determined by a filtration $\left\{B_{i}\right\}$, where $B_{i}=B_{i+1}$ implies that $B_{i}=B_{i+k}$ for each positive integer $k$, is in $B(R)$.

(2) If the sequence $\left\{A_{i}\right\}$ of ideals defined by (1.1) is a filtration and if $\lim _{i \rightarrow \infty} w\left(A_{i}\right)=\infty$, then $w \in B(R)$. Both of these conditions are satisfied if $w$ is a valuation on $R$ and $R$ is noetherian. 
(3) A pseudo-valuation $w$ on $R$ such that the range of $w$ is equal to the set of all multiples of some positive real number $t>0$ is in $B(R)$. This includes all integrally valued homogeneous pseudovaluations $w$ such that there is an $x \in R$ for which $w(x)=1$.

(4) All integrally valued pseudo-valuations and pseudo-valuations on a noetherian ring such that (1.1) forms a filtrations are in $B(R)$.

\section{Proof. (1) Clear.}

(2) Let $w$ and $\left\{A_{i}\right\}$ satisfy the hypothesis of the first statement of (2). Clearly $w(x)=\infty$ implies that $x \in \cap A_{i}$. Let $x \in \cap A_{i}$, then $w(x) \geqq w\left(A_{i-1}\right)$ for each $i$. Since $\lim _{i \rightarrow \infty} w\left(A_{i}\right)=\infty, w(x)=\infty$.

We will now prove the second statement of (2). Let $v$ be a valuation on a noetherian ring $R$. By Remark 1, the sequence of ideals $\left\{A_{i}\right\}$ defined by (1.1) is a filtration in $R$. We need to show that $\lim _{i \rightarrow \infty} v\left(A_{i}\right)=\infty$. Consider a basis $\left\{y_{1}, \cdots, y_{r}\right\}$ for the ideal $A_{1}$. Let $v\left(y_{1}\right)=\min \left\{v\left(y_{1}\right), \cdots, v\left(y_{r}\right)\right\}$. Then $y_{1}$ is an element of $R$ with the property that $v\left(y_{1}\right)=\varepsilon$ is a minimal positive element in the range of $v$. Assume that $\lim _{i \rightarrow \infty} v\left(A_{i}\right)=t<\infty$. By Lemma $3(\mathrm{~b}), v\left(A_{i}\right)>v\left(A_{i-1}\right)$ for each $i$. Thus we can choose a sequence $\left\{x_{j}\right\} \in R$ so that $v\left(x_{j}\right)=a_{j}$ where $(t-\varepsilon)<a_{1}<a_{2}<\cdots$, and each $a_{j}<t$. Let $B$ be the ideal generated by $\left\{x_{j}\right\}$. Since $R$ is noetherian there exists a positive integer $n$ so that $\left\{x_{1}, \cdots, x_{n}\right\}$ is a basis of $B$. Let $p>n$, then $x_{p} \in B$ and so $x_{p}=\sum_{i=1}^{n} \alpha_{i} x_{i}, \alpha_{i} \in R$. Then $v\left(x_{p}\right) \geqq \min \left\{v\left(\alpha_{1} x_{1}\right), \cdots, v\left(\alpha_{n} x_{n}\right)\right\}$. Let $v\left(\alpha_{j} x_{j}\right)$ be this minimum. Case 1: If $v\left(\alpha_{j}\right) \neq 0$, then $v\left(x_{p}\right) \geqq v\left(\alpha_{j}\right)+a_{j} \geqq \varepsilon+a_{i} \geqq t$, which is a contradiction. Case 2: If $v\left(\alpha_{j}\right)=0$, then $v\left(x_{p}\right)=a_{p}>a_{j}=$ $v\left(x_{j}\right)=v\left(\alpha_{j} x_{j}\right)$. By properties of a valuation, $v\left(\alpha_{j} x_{j}\right)=v\left(\alpha_{k} x_{k}\right)$ for some $k \leqq n, k \neq j$. Since $v\left(x_{j}\right) \neq v\left(x_{k}\right), v\left(\alpha_{k}\right) \neq 0$. Hence, $v\left(x_{p}\right) \geqq v\left(\alpha_{k}\right)+a_{k} \geqq t$, a contradiction. This proves that $\lim _{i \rightarrow \infty} v\left(A_{i}\right)=\infty$.

(3) Define $B_{0}=R$ and inductively, $B_{i}=\{x \in R: w(x) \geqq i \cdot t\}$. The sequence $\left\{B_{i}\right\}$ satisfies the hypothesis of Lemma 2 and is a best filtration for $w$.

(4) The first part is clear. For the second part use the same technique as in (2).

2. The main results. The following notation will be used in this section. Let $S$ be an overring of $R$. If $A$ is an ideal of $R$, then the extension of $A$ to $S, A \cdot S$, will be denoted by $A^{e}$. If $B$ is an ideal of $S$, then the contraction of $B$ to $R, B \cap R$, will be denoted by $B^{c}$.

THEOREM 2. Suppose that $S$ is an overring of $R, w_{0} \in B(R)$, and $\left\{A_{i}\right\}$ is the best filtration for $w_{0}$. If each $A_{i}$ is a contracted ideal with respect to $S$, then $w_{0}$ can be extended to $S$.

Proof. Define $B_{i}=A_{i}^{e}$ for each $i$. Then $\left\{B_{i}\right\}$ is a filtration on $S$. 
Define a mapping $w$ on $S$ as follows: $w(x)=w_{0}\left(A_{i}\right)$ if $x \in B_{i}, x \notin B_{i+1}$ and $w(x)=\infty$ if $x \in \cap B_{i}$. We will show that $w$ is a pseudo-valuation on $S$ which extends $w_{0}$ to $S$. Property (i) of the definition of pseudovaluation is obviously satisfied. Suppose that $x \in B_{i}, x \notin B_{i+1}$ and $y \in B_{j}$, $y \notin B_{j+1}$. Without loss of generality, assume that $i \leqq j$. Then $x-y \in B_{i}$ and hence, $w(x-y) \geqq w_{0}\left(A_{i}\right)=\min \left\{w_{0}\left(A_{i}\right), w_{0}\left(A_{j}\right)\right\}=\min \{w(x), w(y)\}$. Similarly if either $x \in B_{i}$ for all $i$ or $y \in B_{j}$ for all $j$, then $w(x-y) \geqq \min \{w(x), w(y)\}$. This proves property (ii).

Finally, we wish to show $w(x y) \geqq w(x)+w(y)$. Again let $x \in B_{i}$, $x \notin B_{i+1}$ and $y \in B_{j}, y \notin B_{j+1}$. Then $x y \in B_{i} B_{j} \subset B_{i+j}$, so that

$$
w(x y) \geqq w_{0}\left(A_{i+j}\right) \text {. }
$$

If $w_{0}\left(A_{i}\right)+w_{0}\left(A_{j}\right) \leqq w_{0}\left(A_{i+j}\right)$, then $w(x y) \geqq w(x)+w(y)$. On the other hand, if $w_{0}\left(A_{i}\right)+w_{0}\left(A_{j}\right)>w_{0}\left(A_{i+j}\right)$, there are two cases to consider. Case 1: Suppose there is a positive integer $t$ such that

$$
w_{0}\left(A_{i}\right)+w_{0}\left(A_{j}\right) \leqq w_{0}\left(A_{i+j+t}\right),
$$

but $w_{0}\left(A_{i}\right)+w_{0}\left(A_{j}\right)>w_{0}\left(A_{i+j+t-1}\right)$. Then $A_{i} A_{j} \subset A_{i+j+t}$, and hence $B_{i} B_{j} \subset B_{i+j+t}$. Since $x y \in B_{i} B_{j} \subset B_{i+j+t}, w(x y) \geqq w(x)+w(y)$. Case 2: Suppose that $w_{0}\left(A_{i}\right)+w_{0}\left(A_{j}\right)>w_{0}\left(A_{i+j+t}\right)$ for all $t$. Then $w_{0}\left(A_{i} A_{j}\right)>w_{0}\left(A_{k}\right)$ for all $k$, which implies that $A_{i} A_{j} \subset A_{k}$ for all $k$. Hence,

$$
x y \in A_{i}^{e} A_{j}^{e} \subset\left(\bigcap_{k=1}^{\infty} A_{k}\right)^{e} \subset \bigcap_{k=1}^{\infty}\left(A_{k}^{e}\right)=\bigcap_{k=1}^{\infty} B_{k} .
$$

Therefore $w(x y)=\infty$ and $w(x y) \geqq w(x)+w(y)$. When either $x \in \cap B_{k}$ or $y \in \cap B_{k}$, clearly $w(x y)=w(x)+w(y)=\infty$. This proves property (iii), showing that $w$ is a pseudo-valuation on $S$.

It is easy to see that $w$ extends $w_{0}$. Take $z \in R$. If $z \in A_{i}, z \notin A_{i+1}$ then by Lemma $1, w_{0}(z)=w_{0}\left(A_{i}\right)$. Clearly $z \in B_{i}$. Suppose $z \in B_{i+1}$, since $z$ is also in $R, z \in A_{i+1}{ }^{e c}=A_{i+1}$ a contradiction. Therefore $z \notin B_{i+1}$, and hence $w(z)=w_{0}\left(A_{i}\right)$. If $z \in \cap A_{i}$, then $z \in \cap B_{i}$ which implies that $w_{0}(z)=w(z)=\infty$.

A subring $R$ of a ring $S$ is said to have property $C$ with respect to $S$ in case each ideal of $R$ is a contraction of an ideal in $S$. In [1], Gilmer shows that in several cases, if $S$ is an overring of $R$ which is integrally dependent on $R$, then $R$ has property $C$ with respect to $S$. Using Gilmer's theory we obtain several applications of Theorem 2 , which are listed in the corollaries below. A Prüfer domain is a domain $R$ with identity in which each finitely generated ideal is invertible, or equivalently, in which $R_{P}$ is a valuation ring for each prime ideal $P$ in $R$. An ideal $A$ of a ring $R$ is called a valuation ideal in case there exists a valuation ring $R_{v}$ containing $R$ and an ideal $B$ of $R_{v}$ such that $B \cap R=A$. 
Corollary 1. Suppose that $R$ is a Prïfer domain with quotient field $K$ and that $R$ is a subdomain of $R_{1}$. If $R_{1} \cap K=R$, then every $w \in B(R)$ can be extended to $R_{1}$.

Proof. By [1; p. 563, Corollary 2], $R$ has property $C$ with respect to $R_{1}$. Then each ideal in a best filtration for $w$ is a contracted ideal with respect to $R_{1}$. By Theorem $2, w$ can be extended to $R_{1}$.

COROLlaRy 2. Let $R$ be a domain, let $w \in B(R)$, suppose that $R_{1}$ is integral over $R$, and let $\left\{A_{i}\right\}$ be the best filtration for $w$. If each $A_{i}$ is an intersection of valuation ideals of $R$, then $w$ can be extended to $R_{1}$.

Proof. Apply [1; p. 564, Th. 2] and Theorem 2.

It is known that if $R$ is an integrally closed domain, $A$ is a complete ideal in $R$ if and only if $A$ is the intersection of valuation ideals. Now let $R$ be an integrally closed domain with quotient field $K, L$ a finite algebraic extension of $K$, and $R^{\prime}$ the integral closure of $R$ in $L$. By [1; p. 569, Th. 6] and Theorem 2, we have:

Corollary 3. If $R^{\prime}$ has an integral basis over $R$ and if $w \in B(R)$, then $w$ can be extended to $R^{\prime}$.

Theorem 3. Suppose that $R$ is a subring of the ring $S$ and suppose that $w_{0}$ is a pseudo-valuation on $R$ which has an extension to a pseudo-valuation $w$ on $S$. If $\alpha$ belongs to the set of extended reals, then the ideals $A_{\alpha}=\left\{x \in R: w_{0}(x)>\alpha\right\}$ and $B_{\alpha}=\left\{x \in R: w_{0}(x) \geqq \alpha\right\}$ are contractions of ideals of $S$.

Proof. $A_{\alpha}$ is the contraction of $A_{\alpha}^{\prime}=\{x \in S: w(x)>\alpha\}$ and $B_{\alpha}$ is the contraction of $B_{\alpha}^{\prime}=\{x \in S: w(x) \geqq \alpha\}$.

The converse of Theorem 2 is also true.

THEOREM 4. Let $S$ be an overring of $R$, let $w_{0} \in B(R)$, and let $\left\{A_{i}\right\}$ be the best filtration for $w_{0}$. If $w_{0}$ can be extended to $S$, then each $A_{i}$ is a contracted ideal with respect to $S$.

Proof. Apply Theorem 3.

Corollary 4. Suppose $w_{0} \in B(R)$ can be extended to some $w$ on $R_{v}$, where $R_{v}$ is a valuation ring. Then each ideal in the best filtration of $w_{0}$ is a valuation ideal.

REMARK 2. Let $R$ be a domain with quotient field $K$ and $w_{0}$ a 
nonnegative pseudo-valuation on $R$. (Nonnegative pseudo-valuations were the most important types of pseudo-valuations studied in [2] and [3]). $w_{0}$ can always be extended to a nonnegative pseudo-valuation $w$ on a subring $R^{\prime}$, where $R \subset R^{\prime} \subset K$, in the following way. Let $M$ be the set of $y \in R$ such that $w_{0}(y)<\infty$ and $w_{0}(x y)=w_{0}(x)+w_{0}(y)$ for all $x \in R$. Then $M$ is a multiplicative subset of $R$ not containing zero. Hence we can form the quotient ring of $R$ with respect to $M, R_{M}$. A function $w^{\prime}$ can be defined on $R_{M}$ by $w^{\prime}(x / y)=w_{0}(x)-w_{0}(y)$. $w^{\prime}$ is not necessarily nonnegative. However, if $R^{\prime}=\left\{z \in R_{M}: w^{\prime}(z) \geqq 0\right\}$ and $w$ is the restriction of $w^{\prime}$ to $R^{\prime}$, then $R^{\prime}$ is a ring and $w$ is an extension of $w_{0}$ to $R^{\prime} . R^{\prime}$ is called the natural domain of $w_{0}$. This type of extension was discussed and used in [2].

I would like to express my gratitude to the referee for his many suggestions on this manuscript. In particular, I would like to thank him for his suggested statement and proof of Theorem 3 .

\section{REFERENCES}

1. Robert W. Gilmer, Jr., Contracted ideals with respect to integral extensions Duke Math. J. 34 (1967), 561-571.

2. James A. Huckaba, Some results on pseudo-valuations (to appear in Duke Math. J.)

3. D. Rees, Valuations associated with a local ring I, Proc. London Math. Soc. (3) 5 (1955), 107-128.

Received June 10, 1968. This work was partially supported by a summer research grant from University of Missouri.

UNIVERSITY OF MISSOURI

Columbia, Missouri 


\section{PACIFIC JOURNAL OF MATHEMATICS}

\section{EDITORS}

H. ROYDEN
Stanford University
Stanford, California

\section{R. R. PHELPS}

University of Washington

Seattle, Washington 98105
J. DUGUNDJI

Department of Mathematics

University of Southern California

Los Angeles, California 90007

\section{RICHARD ARENS}

University of California

Los Angeles, California 90024

\section{ASSOCIATE EDITORS}

E. F. BeCKenbaCh
B. H. NEUMANN

F. WOLF
K. YoshidA

\section{SUPPORTING INSTITUTIONS}

\author{
UNIVERSITY OF BRITISH COLUMBIA \\ CALIFORNIA INSTITUTE OF TECHNOLOGY \\ UNIVERSITY OF CALIFORNIA \\ MONTANA STATE UNIVERSITY \\ UNIVERSITY OF NEVADA \\ NEW MEXICO STATE UNIVERSITY \\ OREGON STATE UNIVERSITY \\ UNIVERSITY OF OREGON \\ OSAKA UNIVERSITY \\ UNIVERSITY OF SOUTHERN CALIFORNIA
}

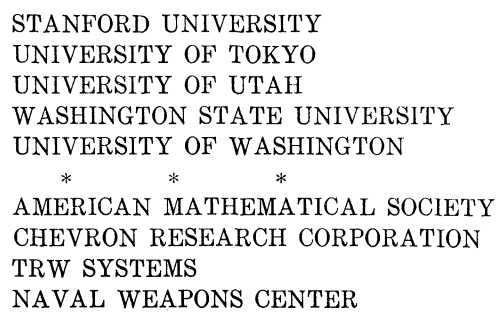

The Supporting Institutions listed above contribute to the cost of publication of this Journal, but they are not owners or publishers and have no responsibility for its content or policies.

Mathematical papers intended for publication in the Pacific Journal of Mathematics should be in typed form or offset-reproduced, double spaced with large margins. Underline Greek letters in red, German in green, and script in blue. The first paragraph or two must be capable of being used separately as a synopsis of the entire paper. It should not contain references to the bibliography. Manuscripts, in duplicate if possible, may be sent to any one of the four editors. Please classify according to the scheme of Math. Rev. 36, 1539-1546. All other communications to the editors should be addressed to the managing editor, Richard Arens, University of California, Los Angeles, California, 90024.

50 reprints are provided free for each article; additional copies may be obtained at cost in multiples of 50 .

The Pacific Journal of Mathematics is published monthly. Effective with Volume 16 the price per volume (3 numbers) is $\$ 8.00$; single issues, $\$ 3.00$. Special price for current issues to individual faculty members of supporting institutions and to individual members of the American Mathematical Society: $\$ 4.00$ per volume; single issues $\$ 1.50$. Back numbers are available.

Subscriptions, orders for back numbers, and changes of address should be sent to Pacific Journal of Mathematics, 103 Highland Boulevard, Berkeley, California, 94708.

PUBLISHED BY PACIFIC JOURNAL OF MATHEMATICS, A NON-PROFIT CORPORATION

Printed at Kokusai Bunken Insatsusha (International Academic Printing Co., Ltd.), 7-17, Fujimi 2-chome, Chiyoda-ku, Tokyo, Japan. 


\section{Pacific Journal of Mathematics \\ Vol. 29, No. $2 \quad$ June, 1969}

Bruce Langworthy Chalmers, On boundary behavior of the Bergman kernel function and related domain functionals ................... 243

William Eugene Coppage, Peirce decomposition in simple Lie-admissible power-associative rings .............................. 251

Edwin Duda, Compactness of mappings...................... 259

Earl F. Ecklund Jr., On prime divisors of the binomial coefficient......... 267

Don E. Edmondson, A modular topological lattice ............... 271

Phillip Alan Griffith, A note on a theorem of Hill ................... 279

Marcel Herzog, On finite groups with independent cyclic Sylow

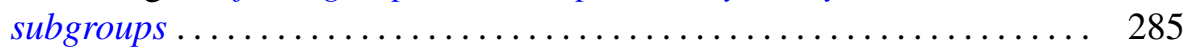

James A. Huckaba, Extensions of pseudo-valuations................. 295

S. A. Huq, Semivarieties and subfunctors of the identity functor ........ 303

I. Martin (Irving) Isaacs and Donald Steven Passman, Finite groups with small character degrees and large prime divisors. II ............ 311

Carl Kallina, A Green's function approach to perturbations of periodic

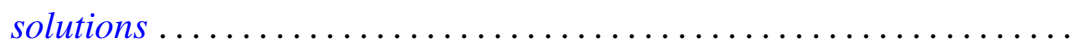

$\mathrm{Al}$ (Allen Frederick) Kelley, Jr., Analytic two-dimensional subcenter manifolds for systems with an integral ....................

Alistair H. Lachlan, Initial segments of one-one degrees ............ 351

Marion-Josephine Lim, Rank k Grassmann products ............. 367

Raymond J. McGivney and William Henry Ruckle, Multiplier algebras of

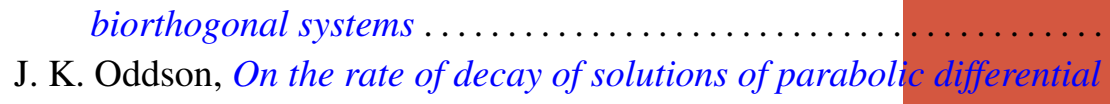

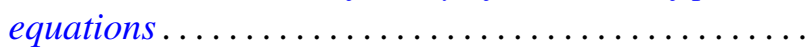

Helmut R. Salzmann, Geometries on surfaces ........... .

Annemarie Schlette, Artinian, almost abelian groups and their groups of automorphisms ............................

Edgar Lee Stout, Additional results on modules over polydisc algebras ...

Lajos Tamássy, A characteristic property of the sphere . .

Mark Lawrence Teply, Some aspects of Goldie's torsion theory. ...

Freddie Eugene Tidmore, Extremal structure of star-shaped sets ...

461

Leon Jarome Weill, Unconditional and shrinking bases in locally convex spaces... 